\title{
Erratum to: Sclerosing Stromal Tumor of the Ovary in Postmenopausal Women: A Report of Two Cases
}

\author{
Chae Min Lee ${ }^{1}$, Soyi Lim ${ }^{1}$, Hyun Yi Cho ${ }^{2}$, Ji-Sung Lee ${ }^{1}$, Jin Woo Shin ${ }^{1}$ \\ ${ }^{1}$ Department of Obstetrics and Gynecology, ${ }^{2}$ Department of Pathology, Gachon University Gil Hospital, Incheon, Korea
}

J Menopausal Med 2015;21(2):115-119

In this article, on page 115, the title has been spelled incorrectly in this article: "Sromal" should have appeared as "Stromal".

The title should be corrected as the following:

from "Sclerosing Sromal Tumor of the Ovary in Postmenopausal Women: A Report of Two Cases"

to "Sclerosing Stromal Tumor of the Ovary in Postmenopausal Women: A Report of Two Cases"

We apologize for any inconvenience caused.

Address for Correspondence: Soyi Lim, Department of Obstetrics and Gynecology, Gachon University Gil Hospital, Namdong-daero 774 beon-gil, Namdong-gu, Incheon 21565, Korea

Tel: +82-32-460-3254, Fax: +82-32-460-3290, E-mail: soyilim@gmail.com 